\title{
Active Vibration Isolation in Ships: A Pre-Analysis of Sound and Vibration Problems
}

\author{
Mathias Winberg, Sven Johansson ${ }^{\dagger}$, Lars Håkansson ${ }^{\dagger}$ and Ingvar Claesson \\ Department of Signal Processing, Blekinge Institute of Technology, S-372 25 Ronneby, Sweden
}

\author{
Thomas Lagö ${ }^{\dagger}$ \\ Axiom EduTech Inc., 70 Himalaya Ct, Alpine, Utah 84004, USA
}

(Received 27 June 2005; accepted 18 October 2005)

\begin{abstract}
Engine-induced sound and vibration levels in boats for professional and leisure use are in many cases unacceptably high in terms of comfort and environmental disturbance. Classical methods for passive treatment are normally less efficient due to the low frequency content and often lead to a substantial increase in weight. The requirements for lower weight, which would increase the maximum speed of the boat as well as improve fuel economy, have to be considered. More efficient vibration damping methods must therefore be found. With, for example, active engine mounts, it is possible to achieve a decrease in the vibrations even for cases when the hull is not very stiff. This is especially important in marine applications, since the engines are usually mounted on flexible and light structures. The project Active Vibration Isolation in Ships (AVIIS) aims at investigating the effects of using a type of Active Noise and Vibration Control system (ANVC) in this particular marine application. This paper presents an analysis of sound and vibration problems in one particular leisure boat from an ANVC point of view. A very thorough investigation was carried out, and the main noise and vibration sources were established as well as the transmission paths of the noise into the boat. Answers were made after this investigation about where the actuators should be positioned, which kind of ANVC approach should be used and the expected interior noise reduction. This is the kind of pre-analysis that is needed for a complex structure, such as that found in a marine vessel, for the successful implementation of ANVC. An optimised passive engine mount, with a stiffness adapted to hull mobility and the engine vibration level, was also designed and evaluated, resulting in an A-weighted saloon sound pressure level reduction of $10 \mathrm{~dB}$ compared to the standard engine mounts. Additionally, with the optimised engine mounts, the vibration levels at the hull were also reduced by up to $15 \mathrm{~dB}$ at the main harmonic components. This paper also presents a feasible way to estimate the performance of a potential active control system based on feedforward narrowband control of engine and propeller harmonics. In this paper secondary sources (inertial mass actuators) are proposed; and for error sensors, accelerometers or microphones, or a combination of the two, are used. In the low frequency range, below $300 \mathrm{~Hz}$, a further reduction of engine orders and propeller BPFs in the order of 5-10 dB are predicted.
\end{abstract}

${ }^{\dagger}$ Member of the International Institute of Acoustics and Vibration (IIAV)

\section{INTRODUCTION}

The engine-induced sound and vibration levels in boats for professional and leisure use are in many cases unacceptably high in terms of comfort and environmental disturbance. In, for instance, the midsize luxury vessel, the Storebro 36 Royal Cruiser, low frequency noise in the saloon (cabin) is considered to degrade the acoustic comfort. In Fig. 1, a schematic view of the Storebro 36 Royal Cruiser is shown. Research carried out at Volvo Penta AB indicates that the noise in the saloon of the Storebro 36 Royal Cruiser is dominated by sound originating from structure-borne noise and that the influence of the directly radiated sound from the engines is insignificant. The dominating sources for mechanical hull excitation and for structure-borne noise are the engines and propellers. Primarily, the engine vibrations propagate via the engine mounts and into the hull. For the propellers, however, there are two alternative transmission paths: either through the water into the hull, or through the propeller shaft into the engine and then through the engine mounts into the hull. Below $400 \mathrm{~Hz}$, the hull excitation sources, the two straight six cylinder turbocharged diesel engines powering the boat and the two propellers, introduce a large number of dominating harmonics in the sound field in the Storebro 36 Royal Cruiser saloon. ${ }^{1}$ These periodic noise components are related to the engine orders and the propeller BPF (Blade Passage Frequency). ${ }^{1}$

The acoustic noise in the boat saloon, induced by the structure-borne noise, may be attenuated for example by reducing the engine and propeller related excitation of the hull. If the hull excitation is dominated by the forces applied by the engine mounts, one way to reduce the noise in the boat saloon would be to reduce the transmissibility of the engine mounts, in other words to reduce the damping and elastic stiffness of the engine mounts. ${ }^{2}$ If the elastic stiffness of an engine mount is too low, however, the transient response of the engine mount system can be a problem and cause shock excitation. Shock excitation can be caused by sudden acceleration and deceleration, such as in the cases of rough sea and running aground. The engine resonance mode in the low frequency range can be excited by such types of excitation. ${ }^{3}$ Furthermore, stiffness values that are too low will lead to large static and quasi-static engine displacements that may 